\title{
FAMILIAL OCCURRENCE IN NEWLY DIAGNOSED EPILEPSY
}

The familial occurrence of epilepsy among 462 children with newly diagnosed multiple unprovoked seizures was studied at the Leiden University Medical Centre, and other hospitals in the Netherlands. Epilepsy was classified as generalized in $57 \%$ of the probands, localization-related in $42 \%$, and undetermined in $1 \%$. Compared to the total group, generalized epilepsies were more common (77\%) and localization-related epilepsies less common $(23 \%)$ in the $10 \%$ of cases found to be familial. Epilepsy occurred in 58 first-degree and 21 other relatives. The majority $(83 \%)$ of first degree relatives with idiopathic or cryptogenic epilepsy had the same seizure type as the proband. (Callenbach PMC, Geerts AT, Arts WFM, et al. Familial occur:ence of epilepsy in children with newly diagnosed multiple seizures: Dutch study of epilepsy in childhood. Epilepsia March 1998;39:331-336). (Reprints: Dr OF Brouwer, Department of Neurology, Leiden University Medical Centre, PO Box 9600, 2300 RC Leiden, The Netherlands).

COMMENT. Epilepsy is familial in $10 \%$ of newly diagnosed cases in childhood. Those with a positive family history are most likely to have generalized, idiopathic or cryptogenic seizures. The relatives of familial cases usually had the same seizure type as the newly diagnosed child. The role of genetic factors in the pathogenesis of idiopathic generalized childhood epilepsy is supported by this study.

\section{LAMOTRIGINE-INDUCED AGGRESSIVE BEHAVIOR}

The response and behavioral profiles of 19 intellectually impaired adults, aged 17-54 years, following treatment with lamotrigine (LTG) as adjunctive therapy for uncontrolled epilepsy are reported from the Epilepsy Research \& Services, Chatswood, Australia. Aggressive behavior and violence were provoked in $9(47 \%)$ patients within less than one month of the introduction of LTG, and 5 required complete withdrawal of the drug. Changes in behavior ranged from shouting, slamming doors, or knocking over furniture to attacks on staff and other residents of the institutions. (Beran RG, Gibson RJ. Aggressive behaviour in intellectually chal lenged patients with epilepsy treated with lamotrigine. Epilepsia March 1998;39:280-282). (Reprints: Dr RG Beran, Epilepsy Research \& Services, Suite 5, 6th Floor, 12 Thomas St, Chatswood NSW 2167, Australia).

COMMENT. The behavior of intellectually impaired patients with epilepsy should be closely monitored when lamotrigine is used as adjunctive therapy for uncontrolled seizures. Violent aggression may be provoked and may require withdrawal of the drug.

\section{MENTAL RETARDATION SYNDROMES}

\section{ANGELMAN SYNDROME WITHOUT CHROMOSOME ANOMALY}

The clinical manifestations of Angelman syndrome (AS) in 12 patients without a cytogenetic or molecular defect and 28 with a deletion were compared at the Department of Neurology, Leiden University Medical Center, and other hospitals in the Netherlands. Two minor differences were uncovered: mandibular prognathism was present in $100 \%$ of patients with a defect and $58 \%$ of those without; truncal hypotonia occurred in only $54 \%$ of the group with a genetic defect and in $91 \%$ of those without. All other characteristics, including seizures and EEG abnormalities, occurred with the same frequency in both groups. The 
absence of a detectable chromosome 15q11-13 anomaly does not change the AS phenotype. (Laan LAEM, Halley DJJ, den Boer ATh, et al. Angelman syndrome without detectable chromosome 15q11-13 anomaly: Clinical study of familial and isolated cases. Am I Med Genet March 1998;76:262-268). (Respond: LAEM Laan MD, Department of Neurology, Leiden University Medical Center, PO Box 9600, 2300 RC Leiden, The Netherlands).

COMMENT. The clinical manifestations of Angelman syndrome with a chromosome defect include delayed motor milestones, ataxic puppet-like gait, absent speech, paroxysmal laughter, mandibular prognathism, macrostomia, brachycephaly, hyperreflexia, truncal hypotonia, and scoliosis. Patients without detectable chromosomal anomaly have a higher frequency of truncal hypotonia and less frequent prognathism. Otherwise the AS phenotype is essentially the same. In a previous study at the University of Pisa, Italy, the puppet-like movements were a manifestation of cortical myoclonus. (Progress in Pediatric Neurology III, 1997;p 390).

\section{RETT SYNDROME, SEIZURES AND NON-SEIZURES}

Prolonged video/EEG/polygraphic monitoring was conducted in 82 Rett syndrome females, ages 2 to 30 years, at Baylor College of Medicine, Houston, TX. Clinical manifestations of reported seizures in $55(67 \%)$ patients included motor symptoms, generalized jerking, stiffening, behavioral changes, and staring. All had abnormal EEGs, epileptiform in 67 (81\%). During monitoring, electrographic seizures were recorded in only $13(16 \%)$ patients, and parents identified the clinical events confirmed as seizures in only 5 . Symptoms considered by parents as typical of 'seizures' were not associated with EEG discharges during monitoring in $23(42 \%)$ of the 55 patients considered initially to have seizures. These 'nonseizures' consisted of twitching, jerking, head turning, falling, trembling, staring, laughing, pupil dilatation, breath holding, and hyperventilation. The occurrence of epileptic seizures in RS is overestimated while true seizures may be under-recognized. (Glaze DG, Schultz RJ, Frost JD. Rett syndrome: characterization of seizures versus non-seizures. Electroencephalogr Clin Neurophysiol Jan 1998;106:79-83). (Respond: Dr Daniel G Glaze. Tel +1 713 7987388; fax +1 713 7984048).

COMMENT. Clinical seizures confirmed by electrographic video monitoring are frequently not recognized as seizures by parents of patients with Rett syndrome, whereas non-seizures are often considered to represent epileptic events. The authors have previously reported correlation of EEG abnormalities in Rett syndrome with the age-related changes in clinical stages. Serial records are characterized by a progressive deterioration, which further confuses the differentiation of seizures and non-seizures. Robb, Harden, and Boyd, at Great Ormond Street Hospital, London, fourd that EEG seizure discharges in 43 of 52 girls with Rett syndrome were not related to the onset of clinical seizures. These and other articles on Rett syndrome are reviewed in Progress in Pediatric Neurology I. 1991;pp513-528.

Growth failure in Rett syndrome was not explained by increased energy expenditure associated with repetitive involuntary movement, in a study of 14 girls at Baylor College of Medicine, Houston. (Motil KJ et al. L Pediatr Feb 1998;132:228-233).

Head circumference at birth in Rett syndrome patients was lower than that for a reference population in a study at the University of Western Australia, Perth. Birth weights were also lower, and $40 \%$ had reported perinatal difficulties and abnormalities in development in the first 6 months. Contrary to 